Bull. Austral. Math. Soc.

VOL. 69 (2004) [237-240]

\title{
EVERY AF-ALGEBRA IS MORITA EQUIVALENT TO A GRAPH ALGEBRA
}

\author{
JASON TYLER
}

\begin{abstract}
We show how to modify any Bratteli diagram $E$ for an AF-algebra $A$ to obtain a Bratteli diagram $K E$ for $A$ whose graph algebra $C^{*}(K E)$ contains both $A$ and $C^{*}(E)$ as full corners.
\end{abstract}

An elegant theorem of Drinen says that every AF-algebra $A$ is isomorphic to a corner in a graph algebra [3, Theorem 1], and hence is Morita equivalent to the graph algebra. The graph in question is a Bratteli diagram for $A$, but it needs to be a carefully chosen one; two constructions of such a diagram were described in [3], one attributed to Kumjian. Here we show that applying Kumjian's construction to an arbitrary Bratteli diagram $E$ for $A$ gives a graph $K E$ whose $C^{*}$-algebra contains both $A$ and $C^{*}(E)$ as full corners, so that $A$ is Morita equivalent to the $C^{*}$-algebra $C^{*}(E)$ of the original Bratteli diagram $E$.

A directed graph $E$ consists of countable sets $E^{0}$ of vertices and $E^{1}$ of edges, along with functions $r, s: E^{1} \rightarrow E^{0}$ which map edges to their range and source vertices. The graph is row-finite if each vertex emits at most finitely many edges. Given a rowfinit graph $E$, a Cuntz-Krieger $E$-family in a $C^{*}$-algebra consists of a set of mutually orthogonal projections $\left\{p_{v}: v \in E^{0}\right\}$ and a set of partial isometries $\left\{s_{e}: e \in E^{\mathbf{1}}\right\}$ satisfying the Cuntz-Krieger relations:

$$
s_{e}^{*} s_{e}=p_{r(e)} \text { for } e \in E^{1} \text { and } p_{v}=\sum_{e \in s^{-1}(v)} s_{e} s_{e}^{*} \text { whenever } s^{-1}(v) \neq \emptyset .
$$

The graph algebra $C^{*}(E)$ is the universal $C^{*}$-algebra generated by a Cuntz-Krieger $E$-family $\left\{s_{e}, p_{v}\right\}$ ([4, Theorem 1.2]). We denote by $E^{*}$ the set of all finite paths in $E$; that is, sequences of edges $\mu_{1} \mu_{2} \ldots \mu_{n}$ such that $r\left(\mu_{i}\right)=s\left(\mu_{i+1}\right)$ for $1 \leqslant i<n$. We include the vertices as paths of length zero. Given $\mu=\mu_{1} \mu_{2} \ldots \mu_{n} \in E^{*}$, define $s_{\mu}:=s_{\mu_{1}} s_{\mu_{2}} \ldots s_{\mu_{n}}$. It follows from [4, Lemma 1.1] that

$$
C^{*}(E)=\overline{\operatorname{span}}\left\{s_{\mu} s_{\nu}^{*}: \mu, \nu \in E^{*}, r(\mu)=r(\nu)\right\} .
$$

Received 15th July, 2003

The author gives his thanks to his indefatigable supervisor Iain Raeburn for guidance throughout this work.

Copyright Clearance Centre, Inc. Serial-fee code: 0004-9727/04 \$A2.00+0.00. 
A Bratteli diagram is a directed graph $E$ such that:

1. $E^{0}$ is the disjoint union of finite sets $\left\{V_{n}\right\}$,

2. every edge with source in $V_{n}$ has range in $V_{n+1}$, and

3. each $v \in E^{0}$ is labelled with a positive integer $d_{v}$ which satisfies

$$
d_{v} \geqslant \sum_{e \in r^{-1}(v)} d_{s(e)}
$$

We say that $E$ is a Bratteli diagram for a sequence of $C^{*}$-algebras $A_{1} \subset A_{2} \subset \ldots$ if each $A_{n}$ is isomorphic to $\underset{v \in V_{n}}{\bigoplus} M_{d_{v}}(\mathbb{C})$ and the embedding of each $M_{d_{v}}(\mathbb{C}) \subset A_{n}$ in each $M_{d_{w}}(\mathbb{C}) \subset A_{n+1}$ scales the trace by $\#\left(s^{-1}(v) \cap r^{-1}(w)\right)$. We say that $E$ is a Bratteli diagram for an AF-algebra $A$ if there exists a sequence of $C^{*}$-subalgebras $\left\{A_{n}\right\}$ of $A$ such that $A=\overline{\cup A_{n}}$ and $E$ is a Bratteli diagram for $\left\{A_{n}\right\}$.

THEOREM 1. Let $E$ be a Bratteli diagram for an AF-algebra $A$. Then there exists a Bratteli diagram $K E$ for $A$ - such that $C^{*}(K E)$ contains $A$ and $C^{*}(E)$ as complementary full corners.

The projection $p$ defining the corner is the sum $p=\sum_{v \in S} p_{\nu}$ where $S \subset K E^{0}$; this sum converges strictly to a projection in $M\left(C^{*}(K E)\right)$ by [1, Lemma 1.1]. Crucial for us is the observation that for $\mu, \nu \in K E^{*}$,

$$
p s_{\mu} s_{\nu}^{*}= \begin{cases}s_{\mu} s_{\nu}^{*} & \text { if } s(\mu) \in S \\ 0 & \text { otherwise }\end{cases}
$$

so that

$$
p C^{*}(K E) p=\overline{\operatorname{span}}\left\{s_{\mu} s_{\nu}^{*}: s(\mu), s(\nu) \in S, r(\mu)=r(\nu)\right\} .
$$

Proof of THE THEOREM: For $n>0$, denote by $V_{n}$ the set of vertices on the $n^{\text {th }}$ level of $E$, and let $V_{0}=\emptyset$. For each $v \in E^{0}$, let $d_{v}$ be the rank of the matrix algebra corresponding to $v$. For every vertex $v \in E^{0}$, calculate $\sigma_{v}:=d_{v}-\sum_{e \in r^{-1}(v)} d_{s(e)}$. We define $K E^{0}=\bigcup_{n=0}^{\infty} K V_{n}$, where

$$
K V_{n}:= \begin{cases}V_{n} & \text { if } \sigma_{v}=0 \text { for all } v \in V_{n+1} \\ V_{n} \cup\left\{w_{n}\right\} & \text { if } \sigma_{v}>0 \text { for some } v \in V_{n+1},\end{cases}
$$

and define $K E^{1}$ to be $E^{1}$ together with, for every $w_{n}$ and $v \in V_{n+1}, \sigma_{v}$ edges from $w_{n}$ to $v$. Denote by $S$ the set $K E^{0} \backslash E^{0}=\cup\left\{w_{n}\right\}$, and set $d_{w}=1$ for all $w \in S$. Constructing $K E$ in this fashion ensures that for all $v \in K E^{0}$, the number of paths beginning in $S$ and ending at $v$ is $d_{v}$. 
Since $E$ is a Bratteli diagram for $A$, there is an increasing sequence of $C^{*}$ subalgebras $F_{n}$ of $A$ such that $A=\overline{\cup F_{n}}$ and $E$ is a Bratteli diagram for the sequence $\left\{F_{n}\right\}$. For those $n$ where $K V_{n} \neq V_{n}$, we define a subalgebra $F_{n}^{\prime}$ of $A$ by $F_{0}^{\prime}:=\mathbb{C} 1$ and

$$
F_{n}^{\prime}:=F_{n} \oplus \mathbb{C}\left(1_{F_{n+1}}-1_{F_{n}}\right) \cong \bigoplus_{v \in V_{n}} M_{d_{v}}(\mathbb{C}) \oplus \mathbb{C} \text { for } n>0 .
$$

For all other $n$, define $F_{n}^{\prime}=F_{n}$. The graph $K E$ is then a Bratteli diagram for the sequence $\left\{F_{n}^{\prime}\right\}$. Since $F_{n} \subseteq F_{n}^{\prime} \subseteq F_{n+1}$ for all $n$, we have $\overline{U F_{n}^{\prime}}=\overline{U F_{n}}=A$; thus $K E$ is a Bratteli diagram for $A$.

Let $\left\{s_{e}, p_{v}\right\}$ be the universal Cuntz-Krieger $K E$-family generating $C^{*}(K E)$. Define a projection $p \in M\left(C^{*}(K E)\right)$ by $p:=\sum_{v \in S} p_{v}$. We aim to show that the corner $p C^{*}(K E) p$ is isomorphic to $A$. Since two algebras with the same Bratteli diagram are isomorphic ([2, Proposition III.2.7]), we can achieve this by identifying a sequence of subalgebras of $p C^{*}(K E) p$ for which $E$ is a Bratteli diagram and whose union is dense in $p C^{*}(K E) p$. For each $n>0$ define $D_{n}:=\operatorname{span}\left\{D^{v}: v \in V_{n}\right\}$, where

$$
D^{\nu}:=\operatorname{span}\left\{s_{\mu} s_{\nu}^{*}: \mu, \nu \in K E^{*}, s(\mu), s(\nu) \in S, r(\mu)=r(\nu)=v\right\}
$$

for each $v \in K E^{0}$. Note that

$$
p C^{*}(K E) p=\overline{\operatorname{span}}\left\{s_{\mu} s_{\nu}^{*}: \mu, \nu \in K E^{*}, s(\mu), s(\nu) \in S, r(\mu)=r(\nu)\right\}=\overline{\cup D_{n}} .
$$

Given $v \in E^{0}$ and paths $\mu, \nu, \alpha, \beta$ with source in $S$ and range $v$, observe that none of $\mu, \nu, \alpha, \beta$ can extend any other since $K E$ contains no loops; [4, Lemma 1.1] then gives

$$
s_{\mu} s_{\nu}^{*} s_{\alpha} s_{\beta}^{*}= \begin{cases}s_{\mu} s_{\beta}^{*} & \text { if } \nu=\alpha \\ 0 & \text { otherwise }\end{cases}
$$

Also, $\left(s_{\mu} s_{\nu}^{*}\right)^{*}=s_{\nu} s_{\mu}^{*}$, so

$$
\left\{s_{\mu} s_{\nu}^{*}: \mu, \nu \in K E^{*}, s(\mu), s(\nu) \in S, r(\mu)=r(\nu)=v\right\}
$$

is a family of matrix units. Since there are $d_{v}$ paths $\mu$ with $s(\mu) \in S$ and $r(\mu)=v, D^{v}$ is isomorphic to $M_{d_{v}}(\mathbb{C})$. Further, note that for distinct $v, w \in V_{n}$, no path ending at $v$ may extend one ending at $w$, so $D^{v} D^{w}=0$ and $D_{n}=\bigoplus_{v \in V_{n}} D^{v} \cong \underset{v \in V_{n}}{\bigoplus} M_{d_{v}}(\mathbb{C})$. It remains only to check that the embedding of each $D_{n}$ in $D_{n+1}$ matches that described by $E$; specifically, for $v \in V_{n}$ and $w \in V_{n+1}$ we need that $D^{v}$ is embedded in $D^{w}$ with multiplicity $\#\left(s^{-1}(v) \cap r^{-1}(w)\right)$. This follows from the Cuntz-Krieger relations at $v$ : 
take paths $\mu, \nu$ with source in $S$ and range $v$, decompose the matrix unit $s_{\mu} s_{\nu}^{*} \in D^{v}$ as

$$
s_{\mu} s_{\nu}^{*}=s_{\mu} p_{\nu} s_{\nu}^{*}=s_{\mu}\left(\sum_{e \in s^{-1}(v)} s_{e} s_{e}^{*}\right) s_{\nu}^{*}=\sum_{e \in s^{-1}(v)} s_{\mu e} s_{\nu e}^{*}
$$

and note that $s_{\mu e} s_{\nu e}^{*}$ is a matrix unit in $D^{w}$ precisely when $e \in r^{-1}(w)$.

Consider now the complementary corner

$$
(1-p) C^{*}(K E)(1-p)=\overline{\operatorname{span}}\left\{s_{\mu} s_{\nu}^{*}: \mu, \nu \in K E^{*}, s(\mu), s(\nu) \in E^{0}, r(\mu)=r(\nu)\right\} .
$$

Since $K E^{1} \backslash E^{1}$ contains only edges from $S$ to $E^{0}$, paths beginning in $E^{0}$ never leave $E^{0}$. Thus $(1-p) C^{*}(K E)(1-p)$ is generated by the Cuntz-Krieger $E$-family

$$
\left\{s_{e}, p_{v}: e \in E^{1}, v \in E^{0}\right\} \text {. }
$$

Further, $E$ contains no loops, so the Cuntz-Krieger uniqueness theorem [1, Theorem 3.1] implies that $(1-p) C^{*}(K E)(1-p)$ is isomorphic to $C^{*}(E)$.

Finally, we must show that $p$ and $1-p$ are full. Note that for every $v \in K E^{0}$ there is a path beginning in $S$ and ending at $v$. Suppose that $I$ is an ideal in $C^{*}(K E)$ containing $p C^{*}(K E) p$; then $I$ certainly contains the projections $\left\{p_{w}: w \in S\right\}$. Given a vertex $v$ in $E^{0}$, choose a path $\alpha$ beginning at some $w \in S$ and ending at $v$. Then $s_{\alpha}=p_{w} s_{\alpha} \in I$, so $p_{v}=s_{\alpha}^{*} s_{\alpha} \in I$, every generator $\left\{s_{e}, p_{v}\right\}$ of $C^{*}(K E)$ is in $I$, and $I=C^{*}(K E)$. Now suppose that $J$ is an ideal in $C^{*}(K E)$ containing $(1-p) C^{*}(K E)(1-p)$, so for every $v \in E^{0}$ we have $p_{v} \in J$. Given a vertex $v \in S$, note that every edge $e$ with $s(e)=v$ satisfies $r(e) \in E^{0}$; so for all $e \in s^{-1}(v)$, we know that $p_{r(e)}=s_{e}^{*} s_{e} \in J$, implying $s_{e}=s_{e} s_{e}^{*} s_{e} \in J$ and $s_{e} s_{e}^{*} \in J$. Thus $p_{v}=\sum_{e \in s^{-1}(v)} s_{e} s_{e}^{*} \in J$, the universal $K E$-family $\left\{s_{e}, p_{v}\right\}$ is contained in $J$, and $J=C^{*}(K E)$.

\section{REFERENCES}

[1] T. Bates. D. Pask, I. Raeburn and W. Szymański, 'The $C^{*}$-algebras of row-finite graphs', New York J. Math. 6 (2000), 307-324.

[2] K. Davidson, $C^{*}$-algebras by example (American Mathematical Society, Providence R.I., 1996).

[3] D. Drinen, 'Viewing $A F$-algebras as graph algebras', Proc. Amer. Math. Soc. 128 (1999), 1991-2000.

[4] A. Kumjian, D. Pask and I. Raeburn, 'Cuntz-Krieger algebras of directed graphs', Pacific J. Math 184 (1998), 161-174.

School of Mathematical and Physical Sciences University of Newcastle

New South Wales 2308

Australia

e-mail: jason.tyler@studentmail.newcastle.edu.au 